\title{
GRANULOMATOSIS WITH POLYANGIITIS: A DIAGNOSTIC CHALLENGE
}

\author{
Bárbara Manfroi ${ }^{1 *}$, Georgia Andressa de Carvalho e Lima Santos ${ }^{1}$, André Marun Lyrio², Tiago Nardi Amaral1, Letícia Maria \\ Kolachinski Raposo Brandão²
}

1. Faculdade de Medicina São Leopoldo Mandic, Campinas (SP), Brazil. 2. Hospital Vera Cruz Campinas, Campinas (SP), Brazil.

*Corresponding author: barbara_manfroi@hotmail.com

\section{BACKGROUND}

Granulomatosis with polyangiitis (GPA) is a necrotizing pauci-immune granulomatous vasculitis that classically affects small vessels, upper and lower respiratory tracts and kidneys. It is a rare disease, with 10:1,000,000 people/year incidence worldwide. Neurological manifestations can occur in 22-54\% of the patients, and may involve central and peripheral nervous system (PNS), which usually manifests with multiple mononeuritis. This report describes a challenging GPA diagnostic case due to the onset of the disease with isolated PNS involvement.

\section{CASE REPORT}

G.K., woman, 51 years old, in follow-up with Neurology team in investigation of decreased sensitivity and paresthesia in her right foot, with acute sensory-motor axonal neuropathy at electroneuromyography. Initially, the patient underwent 5 days of human immunoglobulin intravenous therapy under the hypothesis of Guillain-Barré syndrome, evolving with partial improvement of symptoms.

One month later, she developed right foot drop, purpuric lesions in lower limbs that progressed upwards to the abdomen and upper limbs, in addition to facial acneiform lesions, oral ulcers and hoarseness (without evidence of subglottic stenosis). In few days, she developed asthenia, distal pulp cyanosis of the third left finger, bilateral ocular hyperemia, diplopia and pain in the calves. After exams, infectious conditions and paraneoplastic syndrome were discarded and an evaluation by the Rheumatology team was requested.

There was an increase in acute phase reactants; positive antineutrophil cytoplasmic autoantibodies in the indirect immunofluorescence in high titer; positive antiproteinase-3 antibodies in the enzyme-linked immunosorbent assay; skull resonance without alterations; chest tomography with ground-glass pulmonary opacities in both lungs and laminar pericardial effusion; normal urinalysis. Gathering criteria for GPA, initiated oral corticosteroid therapy with prednisone $1 \mathrm{mg} / \mathrm{kg}$, azathioprine $2 \mathrm{mg} /$ $\mathrm{kg} /$ day orally with almost complete regression of symptoms in 2 weeks, maintaining weakness in the right foot.

Sequentially submitted to half-yearly intravenous therapy with rituximab, progressed with significant clinical and laboratory improvement with sustained remission of the disease until 2 years after diagnosis. During this period, the patient was rigorously submitted to motor rehabilitation with the Physiotherapy team.

\section{CONCLUSION}

The involvement of the peripheral nervous system in vasculitis can represent a diagnostic challenge for neurologists and rheumatologists, especially if presented isolated in the early stages of the disease. In addition, motor rehabilitation proves to be a valuable tool to rescue the functionality and autonomy of patients with PNS involvement. It is concluded that multidisciplinary follow-up must be strongly motivated to optimize early diagnosis and facilitate the rehabilitation of this patient profile. 